DOI: $10.1002 / \mathrm{smll} .201302613$

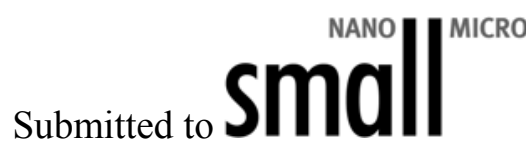

Article type: Full Paper

Accepted for publication September 9, 2013

\title{
Interactions and Chemical Transformations of Coronene Inside and Outside Carbon
} Nanotubes

Bea Botka, Melinda E. Füstös, Hajnalka M. Tóháti, Katalin Németh, Gyöngyi Klupp, Zsolt Szekrényes, Dorina Kocsis, Margita Utczás, Edit Székely, Tamás Váczi, György Tarczay, Rudi Hackl, Thomas W. Chamberlain, Andrei N. Khlobystov, and Katalin Kamarás*

Bea Botka, Dr. Rudi Hackl

Walther-Meissner-Institute, Bavarian Academy of Sciences and Humanities

Walther-Meissner-Strasse 8, 85748 Garching, Germany

Melinda E. Füstös

Faculty of Chemistry and Chemical Engineering

Babeş-Bolyai University

Arany János Str. 11, 400084 Cluj-Napoca, Romania

Hajnalka M. Tóháti, Katalin Németh, Dr. Gyöngyi Klupp, Zsolt Szekrényes, Dorina Kocsis, Prof. Katalin Kamarás

Institute for Solid State Physics and Optics

Wigner Research Centre for Physics

Hungarian Academy of Sciences

P.O.Box 49, 1525 Budapest, Hungary

E-mail: kamaras.katalin@wigner.mta.hu

Margita Utczás, Dr. Edit Székely

Department of Chemical and Environmental Process Engineering

Budapest University of Technology and Economics

Budafoki út 8, 1111 Budapest, Hungary

Dr. Tamás Váczi

Department of Mineralogy

Eötvös Loránd University

Pázmány Péter sétány 1/C, 1117 Budapest, Hungary

Dr. György Tarczay

Laboratory of Molecular Spectroscopy

Institute of Chemistry

Eötvös Loránd University

1518 Budapest, Hungary

Dr. Thomas W. Chamberlain, Prof. Andrei N. Khlobystov

School of Chemistry

University of Nottingham

University Park, Nottingham NG7 2RD, United Kingdom

Keywords: coronene; graphene nanoribbons; carbon nanotubes; encapsulation 


\section{Submited to Small}

By exposing flat and curved carbon surfaces to coronene, a variety of van der Waals hybrid heterostructures were prepared, including coronene encapsulated in carbon nanotubes, and coronene and dicoronylene adsorbed on nanotubes or graphite via $\pi-\pi$ interactions. The structure of the final product is determined by the temperature of the experiment and the curvature of the carbon surface. While at temperatures below and close to the sublimation point of coronene, nanotubes with suitable diameters are filled with single coronene molecules, at higher temperatures additional dimerization and oligomerization of coronene occurs on the surface of carbon nanotubes. The fact that dicoronylene and possible higher oligomers are formed at lower temperatures than expected for vapor-phase polymerization indicates the active role of the carbon surface used primarily as template. Removal of adsorbed species from the nanotube surface is of utmost importance for reliable characterization of encapsulated molecules: it is demonstrated that the green fluorescence attributed previously to encapsulated coronene is instead caused by dicoronylene adsorbed on the surface which can be solubilized and removed using surfactants. After removing most of the adsorbed layer, a combination of Raman spectroscopy and transmission electron microscopy was employed to follow the transformation dynamics of coronene molecules inside nanotubes.

\section{Introduction}

The quest to direct the bottom-up synthesis of graphene and graphene-like structures is at the forefront of chemistry today and despite some encouraging breakthroughs, still contains many challenges. ${ }^{[1,2]}$ Hybrid materials composed of carbon nanotubes and polycyclic aromatic hydrocarbons (PAHs) became of interest recently as the possibility of forming graphene nanoribbons (GNRs) from these systems emerged. An especially attractive member of the PAH family is coronene, because its size is close to the diameter of the most widely studied 


\section{saminasus small}

single-walled carbon nanotubes (SWNTs) and its position within the nanotube can be finetuned by the nanotube diameter. Flat PAH molecules can either be positioned with their plane perpendicular ("stacks") or parallel ("ribbons") to the long axis of the nanotubes. Coronene has been reported to form stacks inside some nanotubes ${ }^{[3]}$ and ribbons in others, ${ }^{[4-7]}$ the latter being susceptible to polymerization ${ }^{[5,8]}$ and thereby GNR formation. Very recently it was reported that annealing coronene-filled nanotubes at high temperature can lead to doublewalled nanotubes (DWNTs). ${ }^{[9,10]}$ Also, because both species possess extended $\pi$-electron networks, adhesion of coronene to the external surface of the nanotube through $\pi-\pi$ interaction is very probable. All of these examples belong to the same class of van der Waals heterostructures, where the constituents are held together by weak interactions and are therefore easy to modify by external stimuli.

An important observation, which has led to the proposal of these materials for a variety of optical applications, is the shift of fluorescence from blue to green of coronene when exposed to nanotubes at $450{ }^{\circ} \mathrm{C}^{[3]}$ While it is known that coronene in similar aggregated states can exhibit such behavior, ${ }^{[1]}$ the exact origin of this fluorescence shift is still to be identified. Two parameters play primary roles in the reactions of coronene in the presence of nanotubes: the reaction temperature and the diameter of the nanotubes. Here we present a study of coronene-carbon nanotube hybrid formation where we systematically varied these two parameters. We find that even in small-diameter nanotube samples, where filling is inhibited, there is strong adhesion to the external nanotube surface via $\pi-\pi$ interaction. Above the sublimation temperature of coronene, templated oligomerization occurs on the surface, ${ }^{[5]}$ yielding mainly the dimeric form dicoronylene. Dicoronylene is insoluble in most solvents but can be brought into suspension with surfactants where it exhibits green fluorescence. We observe surface-enhanced Raman scattering due to the interaction between dicoronylene and the external carbon nanotube surface. The transformations induced in the coronene molecules ${ }^{[5]}$ inside the nanotubes were followed by Raman spectroscopy on thermal annealing 


\section{suminats small}

and by transmission electron microscopy (TEM) on exposure to the electron beam. We conclude that these reactions start from coronene molecules and their final products (at $1250^{\circ} \mathrm{C}$ or for prolonged e-beam exposure) are carbon nanotubes formed from coronene inside the original nanotubes. Intermediate products are identified as polymeric structures, including hydrogenated GNRs.

\section{Results and Discussion}

\subsection{Samples}

We selected carbon nanotubes with as large a range of internal diameters as possible. For reference to study coronene adhesion without encapsulation, we also conducted experiments on highly ordered pyrolytic graphite (Sample GR). A sample of coronene nanowires (Sample CW), which consists of coronene stacks in a face-to-face arrangement, ${ }^{[11]}$ was also used as a reference. Details of the samples are given in Table 1. Theoretical considerations by Okazaki et al. ${ }^{[3]}$ give $1.32 \mathrm{~nm}$ as the minimum diameter for a carbon nanotube which can encapsulate coronene molecules. Based on these calculations, coronene cannot fit inside the majority of the narrowest SWNT sample, CoMoCat (Sample CM). In contrast, the cavity of the multiwalled nanotubes (MWNT; Sample MW) is sufficiently large to allow any orientation, motion and subsequent chemical reactions of coronene molecules to take place. These nanotube samples, nevertheless, are above the diameter where regular graphene nanoribbon formation is possible. ${ }^{[12]}$ P2-SWNT nanotubes, a widely studied SWNT type (Samples SC, LT and HT), can be filled with coronene molecules, but polymerization reactions inside the nanotube may be constrained for steric reasons.

The effect of reaction temperature was studied for P2-SWNT samples. We employed three reaction temperatures: $50{ }^{\circ} \mathrm{C}$ with nanoextraction ${ }^{[13,14]}$ from supercritical $\mathrm{CO}_{2},{ }^{[15]} 385{ }^{\circ} \mathrm{C}$, slightly above the sublimation temperature of coronene, and $450{ }^{\circ} \mathrm{C}$ where coronene was 


\section{s.bminted s small"}

reported to undergo polymerization reactions. ${ }^{[5,8]}$ Investigation of the curvature and diameter dependence was conducted using carbon surfaces with increasing curvature in the sequence graphite $<$ MWNT $<$ P2-SWNT $<$ CoMoCat-SWNT.

Excess coronene and dicoronylene from the nanotube surface was removed by processing the coronene-nanotube hybrid structures. The importance of this step was emphasized before by Gao et al. for oligothiophene-encapsulated nanotubes. ${ }^{[16]}$ For this purpose, we employed repetitive washing with toluene and sodium dodecyl sulfate (SDS) aqueous solution, respectively. The coronene/dicoronylene content of the filtrate was determined by UV/visible spectroscopy and/or fluorescence spectroscopy. As the removal of excess starting coronene constitutes an important part of the established protocol, toluene washing was performed for all samples, apart from those specifically referred to as "as-prepared".

All samples were characterized using a range of different techniques, and their evolution during thermal annealing and exposure to the electron beam was investigated. Initially we will discuss samples prepared from the same nanotubes at different temperatures, then discuss samples prepared at the same temperature but with different carbon nanostructures.

\subsection{Effect of reaction temperature on P2-SWNT based hybrids}

TEM imaging of the P2-SWNTs treated with coronene at different temperatures reveals different structures (Figure 1). The nanotubes filled by nanoextraction at $50{ }^{\circ} \mathrm{C}$ or sublimation at $385{ }^{\circ} \mathrm{C}$ both show coronene stacks similar to those reported by Okazaki et al. ${ }^{[3]}$ Surprisingly the nanoextraction method yielded well-ordered stacks of coronene even at the relatively low temperature. In contrast, sublimation filling at $450{ }^{\circ} \mathrm{C}$ results in considerably fewer coronene stacks with a larger proportion of other types of carbon structures formed inside the host nanotube, including nanotube within nanotube structures.

Raman spectroscopy can be used in many ways for the characterization of nanotube derivatives: the radial breathing mode (RBM) frequency scales inversely with nanotube 

diameter, ${ }^{[17]}$ and both encapsulated and external species can show Raman activity. Raman spectra of the P2-SWNT based samples together with those of reference materials for comparison are shown in Figure 2. (The spectrum of coronene (Figure 2a) was taken with low frequency excitation because of intense photoluminescence at visible wavelengths. The full Raman spectra are shown in Figure S1 of the Supporting Information.) Coronene (Figure 2a) and dicoronylene (Figure 2b) can be clearly distinguished based on their Raman spectra. Comparing all three P2-SWNT based materials, neither coronene nor dicoronylene is detected in the samples prepared at $50^{\circ} \mathrm{C}$ or $385^{\circ} \mathrm{C}$ (samples SC and LT). High temperature treatment at $450{ }^{\circ} \mathrm{C}$, however, results in Raman bands of dicoronylene, but not coronene. Previously it has been reported for $\mathrm{C}_{60} @$ SWNT structures that the Raman lines of the fullerene molecules encapsulated in the nanotubes are rather weak. ${ }^{[18,19]}$ Therefore the high intensity of dicoronylene modes indicates that the observed signal is due to the surface adsorbed dicoronylene molecules rather than those encapsulated inside nanotubes. TEM images give strong corroboration to this hypothesis.

Photoluminescence (PL) measurements were carried out in aqueous SDS suspension. ${ }^{[20]}$ SDS was found to help to transfer dicoronylene into solution, although the exterior of the nanotubes cannot be cleaned completely. The green luminescence of the material prepared by high-temperature vapor filling was reported ${ }^{[3]}$ and attributed to stacks of coronene inside the nanotubes. Our results, summarized in Figure 3, however, do not support this suggestion. Sample SC possesses ordered stacks of coronene inside nanotubes but exhibits no green luminescence. The spectrum also differs from that of coronene nanowires (sample CW), a model for the stacks of coronene without the nanotube. Sample HT, on the other hand, shows a luminescence spectrum identical to that of dicoronylene, markedly different from that of coronene, ${ }^{[21]}$ thus indicating that formation of dicoronylene occurred on the nanotube surface at higher temperature. The suspension of sample LT shows fluorescence bands of both coronene and dicoronylene, but the lack of dicoronylene Raman features in the solid (Figure 


\section{sumers small}

2d) suggests that the dicoronylene content is much lower than that of the HT sample, such that it could be removed by the surfactant in this case.

Our approach is similar to two previous studies: Muramatsu et al. ${ }^{[22,23]}$ reported strong

luminescence from the inner tubes of peapod-derived DWNTs. However, later results ${ }^{[24,25]}$ showed that this luminescence may result from unencapsulated inner tubes produced by sonication when preparing the suspension for PL measurements, a similar conclusion to our observations. Loi et al. ${ }^{[16,26]}$ encapsulated small oligothiophenes into carbon nanotubes and studied them with similar methods. Here the authors succeeded in ruling out the presence of any unencapsulated species, and found that the emission spectrum changes little upon encapsulation. In the present study, however, in some of our hybrid materials we observe strong luminescence which is distinctly different from the coronene spectrum, and which we assign to dicoronylene, dissolved from the nanotube surface into the aqueous surfactant solution.

\subsection{Effect of curvature of carbon surface}

Figure 4 shows TEM images of nanotubes with internal diameters narrower (CoMoCatSWNT) and wider (MWNT) than P2-SWNTs. As expected, the cavities of the narrow CoMoCat nanotubes are empty and do not contain either coronene or other guest species. In the large cavities of MWNTs, on the other hand, signs of dimerization and oligomerization of coronene are clearly observed.

Raman spectra of samples HT, CM, MW and GR, along with those of the corresponding reference materials, are shown in Figure 5. All samples prepared at high temperature (including the small-diameter CoMoCat nanotubes and graphite) exhibit dicoronylene spectra similar to sample HT. Since the presence of dicoronylene appears to be independent of the encapsulation of coronene, we attribute the dicoronylene spectrum to adsorbed molecules on the outer surfaces of carbon nanostructures. 


\section{submitud to Small}

The as-prepared material from high-temperature synthesis contains both coronene and dicoronylene adhered to the surface. Coronene can be removed by toluene washing (see Figure S2 in the Supporting Information), whilst further SDS treatment removes mostly dicoronylene. The Raman spectrum of the as-prepared sample SC, which contains no dicoronylene, indicates the presence of coronene which disappears after toluene treatment (Figure 6a). In contrast, Figure 6b illustrates that the intensity of the dicoronylene bands, normalized to the G band of the nanotubes, hardly changes after SDS washing. An explanation for this constant intensity would be that the first layer of dicoronylene in the immediate vicinity of the nanotube sidewall dominates the Raman spectra due to surfaceenhanced scattering. The evanescent field necessary for surface enhancement is provided by the delocalized $\pi$-electron system of the nanotubes; surface attenuation of infrared spectra of adsorbed molecules has been explained by a similar mechanism. ${ }^{[27,28]}$

Aqueous surfactant suspensions used for photoluminescence measurements in this study as well as in previous published works, ${ }^{[3,4,6,7]}$ may contain, beside nanotube-centered micelles, dissolved excess dicoronylene from the external surface of the nanotubes. To determine the origin of the emission, we took photoluminescence spectra of the filtered solutions which contained almost no nanotubes. The toluene solution contains only coronene, but a large amount of dicoronylene is present in the aqueous SDS solution after washing (Figure 6c). Photographs of filtrate SDS solutions in UV light $(365 \mathrm{~nm})$ after washing the samples (Figure 6d) further substantiate the claim that the fluorescence in SDS solution, observed previously, originates from the excess adsorbed dicoronylene suspended by the surfactant rather than the encapsulated coronene. Figure $6 \mathrm{~d}$ shows the first and 3rd filtrate, the former showing much stronger fluorescence than the latter.

The photoluminescence spectra of samples HT and CM in suspended in aqueous SDS solution proved to be identical to each other and to that of dicoronylene (Figure 7). To deduce whether this green photoluminescence comes from the molecules remaining in contact with the 


\section{sandisus small}

suspended nanotubes or those desorbed in the solution, we prepared a suspension of the CoMoCat nanotube-coronene hybrid (Sample CM) in aqueous SDS which was subsequently filtered. The photoluminescence spectra of both the nanotube suspension and the filtrate were measured at various excitation wavelengths. Figure 8 shows the photoluminescence results for the near-infrared (NIR) and visible region. The NIR luminescence excitation map is typical of the CoMoCat sample, ${ }^{[29,30]}$ with nanotubes of $(6,5)$ chirality forming the majority. In addition, dicoronylene emission is seen at $356 \mathrm{~nm}$ excitation. In the filtrate, the latter emission remains while the NIR luminescence disappears. These results demonstrate that the bulk of the emission originates from the dissolved dicoronylene, not from the molecules in contact with the nanotubes, unlike the Raman spectra of solid samples which unambigously show the presence of adsorbed dicoronylene.

\subsection{Transformations of coronene inside nanotubes: Effect of annealing and electron irradiation}

As mentioned above, the presence of encapsulated coronene can be confirmed by annealing the hybrid structures to form DWNTs and detecting the inner-nanotube RBM modes in the Raman spectra. ${ }^{[10]}$ We chose samples SC and LT to follow the thermal evolution of the molecules in the nanotube cavities, because these two materials have little or no adsorbed dicoronylene on the nanotube surface which can potentially obscure the observation of encapsulated molecules. Figure 9 shows Raman spectra for both samples. The effect of annealing at 500 and $700{ }^{\circ} \mathrm{C}$ is the appearance of a complex pattern around $1400 \mathrm{~cm}^{-1}$ in both cases, similar to those reported in Ref. 8 for coronene oligomers. For annealing temperatures between 900 and $1250{ }^{\circ} \mathrm{C}$ the bands in the range $1300-1450 \mathrm{~cm}^{-1}$ disappear while the inner-nanotube radial breathing modes ${ }^{[31,32]}$ appear. These are the results of the thermally induced reaction of the polycyclic aromatic hydrocarbon molecules inside the nanotubes, ultimately leading to the formation of inner nanotubes. Such reactions have been 


\section{Subitited to Small"}

reported for encapsulated fullerenes, ${ }^{[33]}$ fullerene-solvent mixtures ${ }^{[34]}$ and ferrocene, ${ }^{[35]}$ inside nanotubes. The observed transformation is likely to have a stepwise mechanism via polymerization of the coronene within the nanotubes. Our TEM observations indicate that the reaction can start from a stacked arrangement of coronene, even though the mutual orientations of molecules within the stack are unfavorable for direct dimerization and polymerization. The heat treatment process may disturb the stacks and thus align the molecules for more facile reactions leading to nanoribbons. This starting point is in contrast to the model presented in Ref. 5, where dicoronylene molecules are supposed to form during sublimation of coronene and these dimers would be encapsulated by the nanotubes. The energetics of such a process is unknown, however it is likely that the strength of the adhesion of dicoronylene via $\pi-\pi$ interactions to the nanotube surface is at least comparable, if not superior, to the energy profile of the rather bulky dicoronylene molecule entering the nanotube cavity. Intuitively, it is more plausible that the coronene molecules enter the tubes individually and depending on their kinetic energy (determined by temperature) undergo further rearrangement and chemical reactions. During the course of polymerization, hydrogenated graphene nanoribbons may form at specific temperatures. However, for a double-walled nanotube to form (which obviously happens at $1250^{\circ} \mathrm{C}$ ), further competing and complex reactions must take place. The details of such reactions are not clear at present. A most insightful study of chemical reactions inside nanotubes has been performed by Koshino et al. ${ }^{[36]}$ on encapsulated fullerenes. If we take the analogy with the mechanism found there, we might envision an associative rather than a dissociative mechanism (i.e. disintegration of coronene into atoms, which requires a large activation energy, is not needed). The presence of at least one intermediate phase is also observed during the transformation of $\mathrm{C}_{60} @$ SWNTs into DWNTs. ${ }^{[37]}$ Intermediate products at lower temperatures and/or intermediate electron doses in our case may consist of polymeric forms including graphene nanoribbon-like structures; however, microscopic and spectroscopic results from 


\section{suminus smänall'}

either our measurements presented here or from the literature, do not allow these structures to be unambiguously assigned at present.

We also measured the PL spectra of both series with various excitation frequencies and present the results in Figure S3, Supporting Information. From the spectra it is apparent that for the SC sample, where no dicoronylene is formed during encapsulation, simple toluene washing is enough to remove the luminescence, in this case caused by coronene. For the LT sample, which contains a small amount of adsorbed dicoronylene beside coronene, traces of dicoronylene can be detected even after annealing to $500{ }^{\circ} \mathrm{C}$. Samples annealed to $700{ }^{\circ} \mathrm{C}$ and above do not show any luminescence above the background. Previous studies ${ }^{[3,5]}$ report annealing at $300{ }^{\circ} \mathrm{C}$ in order to remove adsorbed molecules. It seems that after this treatment a substantial amount of adsorbed species can remain and therefore the reported PL and Raman spectra cannot be unambiguously assigned to encapsulated species. The weak Raman bands in Figure 9, in contrast, must come from inside the nanotubes and can be directly compared to those measured by Talyzin et al. ${ }^{[8]}$ for pristine coronene during polymerization. The development of the spectra with temperature shows the same general trends, but no details can be discerned at the given resolution. The $900{ }^{\circ} \mathrm{C}$ spectrum shows a pronounced $\mathrm{D}$ band around $1300 \mathrm{~cm}^{-1}$, indicating a disordered structure before rearrangement into an internal nanotube.

It was shown previously ${ }^{[12]}$ that exposure to the electron beam in TEM leads to similar transformations as thermal treatment. It is therefore interesting to compare the behavior of the samples under prolonged exposure to the electron beam. In addition, for samples coated by dicoronylene (HT, CM and MW) this is the only way to follow reactions inside the nanotubes since the absorption and photoluminescence of dicoronylene on nanotube surfaces hinders the observation by spectroscopic methods. (For the HT sample, the final product DWNT could be identified by the RBM mode which does not overlap with the Raman spectrum of dicoronylene. ${ }^{[10]}$ ) 
Our observations (Figure 1 and 4) indicate that prolonged electron beam exposure produces internal nanotubes in P2-SWNT based samples SC, LT and HT, similar to those observed after annealing at $1250^{\circ} \mathrm{C}$. In contrast, under the same electron beam conditions dimerization of coronene is observed in MWNTs. This difference can be attributed to competition between the templating effect of the inner nanotube wall to form a second internal tube and the tendency of the coronene molecules towards polymerization, with the outcome determined by the size of the nanotube cavity. ${ }^{[12]}$ Coronene dimers are difficult to form within SWNTs due to the severe space restriction inhibiting the coplanar arrangement of molecules required for the reaction. However, the wide space in MWNT cavities allows the molecules to adopt orientations suitable for dimerization/polymerization reactions in the edge-to-edge manner, while the extended gap between MWNT sidewalls may inhibit transformations of coronene into an inner nanotube.

We summarize our observations and their interpretation for the samples in Table 2.

\section{Conclusion}

While systematically varying the synthesis parameters of carbon-coronene hybrids, we prepared a series of encapsulated and adsorbed structures and characterized them by TEM imaging and Raman and PL spectroscopy. We found that the stacked vs. ribbon arrangement of coronene molecules in SWNTs depends on the reaction temperature: filling from supercritical $\mathrm{CO}_{2}$ produces ordered stacks, while vapor-phase filling results in a mixture of arrangements with less stacked molecules as the reaction temperature is increased. Hightemperature vapor filling results in dimerization of coronene and subsequent adhesion of molecules to the external surface of the nanotube. Carbon surfaces tend to facilitate coronene dimerization and form dicoronylene above the sublimation temperature of coronene. The interaction between dicoronylene and the surface might be exceptionally strong, proven by the difficulty in removal and the surface enhancement seen in Raman spectra. The green 


\section{suminas small}

fluorescence of aqueous SDS suspensions of coronene-filled nanotubes originates from dicoronylene molecules dissolved in the SDS solution, as proven by the comparison of nanotube-containing suspensions and the nanotube-free filtrate. Annealing or e-beam exposure of coronene-filled single-walled nanotubes with diameters close to the size of coronene results in the formation of internal nanotubes through complicated polymerization steps, which might involve dehydrogenated nanoribbon formation as intermediate structures; the same treatment in multiwalled nanotubes yields polymerized coronene products and no internal nanotubes. Certainly, analogous hybrids from other polyaromatic hydrocarbons deserve further attention in order to produce regular carbon structures within nanotubes.

\section{Experimental Section}

Sample preparation: For reference and starting material, P2-SWNTs (Carbon Solutions, diameter range: 1.2-1.6 nm); CoMoCat CG (Southwest Nanotechnologies, diameter range 0.5 $-1.2 \mathrm{~nm})^{[38]}$ and multiwalled nanotubes (Nanocyl) were used. Coronene (Sigma-Aldrich, 97\%) and dicoronylene (Kentax $\mathrm{GmbH}, 99.5 \%$ ) were also purchased from commercial sources. Before filling, the starting materials were opened by annealing at $570{ }^{\circ} \mathrm{C}$ in air for 20 minutes. Vapor filling was performed at $450{ }^{\circ} \mathrm{C}$ ("high temperature") and $385{ }^{\circ} \mathrm{C}$ ("low temperature") using the method described by Okazaki et al. ${ }^{[3]}$ (Coronene sublimes above 325 ${ }^{\circ} \mathrm{C}$ at $10^{-4}$ mbar.) The nanoextraction ${ }^{[39]}$ was performed in a closed high pressure reactor ${ }^{[18]}$ under supercritical $\mathrm{CO}_{2}$ at $50{ }^{\circ} \mathrm{C}$ and 150 bar for 96 hours. Each sample was repeatedly washed with toluene to remove excess coronene from the exterior of the tubes. The washing process was monitored by UV/visible and/or photoluminescence spectroscopy, until a negligible amount of coronene remained in the toluene filtrate (see Figure S2 in the Supporting Information). Removal of dicoronylene was performed by washing with 1 wt \% aqueous SDS solution. In this case, the last washing SDS solution (after 3 steps) still contained some dicoronylene ( 0.5 per cent of the starting fluorescence intensity). Annealing 


\section{S.tunitats small}

of the encapsulated species was performed in vacuum for 24 hours. In all cases, pristine SWNTs were used as reference; these samples underwent the same annealing sequence without adding coronene.

Transmission electron microscopy: Samples were prepared via drop-drying methanolic solutions onto copper TEM grid mounted "lacey" carbon films. Transmission electron microscopy was performed using a JEOL 2100F TEM (field emission gun source, information limit $<0.19 \mathrm{~nm}$ ) at room temperature and an accelerating voltage of $100 \mathrm{kV}$. Coronene molecules are stable under these conditions for 5-10 seconds which was sufficient for capturing images of coronene stacks in nanotubes. Further exposure of the e-beam results in rapid transformations of coronene into amorphous carbon and internal nanotubes. Image processing and analysis was conducted using Gatan DigitalMicrograph software.

Raman microscopy: Data were acquired in backscattering geometry under ambient conditions. The laser power was carefully adjusted to avoid any type of changes in the samples. Spectra were taken with four different instruments using five excitation lines: 482, 531, $\left(\mathrm{Kr}^{+}\right), 532$ (frequency-doubled 1064), $785 \mathrm{~nm}$ (diode laser) and $1064 \mathrm{~nm}$ (Nd:YAG). The spectral resolution was better than $2 \mathrm{~cm}^{-1}$ in all cases.

UV/VIS spectroscopy: Spectra of toluene solutions were measured by a fiber optics spectrometer (Ocean Optics QE65000) using a deuterium and a halogen light source. Spectral resolution was $1 \mathrm{~nm}$.

Photoluminescence: Fluorescence spectra were taken with a HORIBA Jobin Yvon Fluorolog3 spectrofluorometer (Nanolog). The samples (approx. $1 \mathrm{mg}$ each) were dispersed for 1 hour in $\mathrm{D}_{2} \mathrm{O}(25 \mathrm{~mL})$, containing $1 \mathrm{wt} \% \mathrm{SDS}$ using an ultrasonic bath. Subsequently each solution was centrifuged at $10000 \mathrm{~g}$ for 1 hour and the supernatant was used for measuring. We applied excitation wavelength $356 \mathrm{~nm}$, to make the comparison with Ref. 3 straightforward, although this is not the maximum of the absorption curve. We also used excitation at $468 \mathrm{~nm}$. The integration time was $0.2-1 \mathrm{~s}$. The PL excitation mapping in the NIR range was obtained by 


\section{sumatus small}

integration for $20 \mathrm{~s}$, and individual spectra shown in Figure 8 were taken with integration time $60 \mathrm{~s}$.

\section{Supporting Information}

Supporting Information is available online from the Wiley Online Library or from the author.

\section{Acknowledgements}

Financial support was provided by the European Commission through the ITN Project FINELUMEN (PITN-GA-2008-215399) and the Hungarian National Research Fund (OTKA grants 75813 and 105691) and the Baross Gábor Programme (contract no. REG-KM-09-12009-0044). M.E.F. was supported by the SECTORAL OPERATIONAL PROGRAMME FOR HUMAN RESOURCES DEVELOPMENT 2007-2013, co-financed by the European Social Fund, under the project number POSDRU 107/1.5/S/76841 with the title "Modern Doctoral Studies: Internationalization and Interdisciplinarity". A.N.K. acknowledges financial support of the European Research Council and the Royal Society, and access to TEM enabled by the Nottingham Nanoscience and Nanotechnology Centre.

Received: ((will be filled in by the editorial staff))

Revised: ((will be filled in by the editorial staff)) Published online: ((will be filled in by the editorial staff))

[1] J. Cai, P. Ruffieux, R. Jaafar, M. Bieri, T. Braun, S. Blankenburg, M. Muoth, A. P.

Seitsonen, M. Saleh, X. Feng, K. Müllen, R. Fasel, Nature 2010, 466, 470.

[2] L. Chen, Y. Hernandez, X. Feng, K. Müllen, Angew. Chem. Int. Ed. 2012, 51, 7640.

[3] T. Okazaki, Y. Iizumi, S. Okubo, H. Kataura, Z. Liu, K. Suenaga, Y. Tahara, M. Yudasaka, S. Okada, S. Iijima, Angew. Chem. Int. Ed. 2011, 50, 4853. 
[4] A. V. Talyzin, I. V. Anoshkin, A. V. Krasheninnikov, R. M. Nieminen, A. G. Nasibulin,

H. Jiang, E. I. Kauppinen, Nano Lett. 2011, 11, 4352.

[5] M. Fujihara, Y. Miyata, R. Kitaura, O. Nishimura, C. Camacho, S. Irle, Y. Iizumi,

T. Okazaki, H. Shinohara, J. Phys. Chem. C 2012, 116, 15141.

[6] P. V. Fedotov, A. I. Chernov, A. V. Talyzin, I. V. Anoshkin, A. G. Nasibulin, E. I.

Kauppinen, E. D. Obraztsova, J. Nanoelectron. Optoelectron. 2013, 8, 16.

[7] A. I. Chernov, P. V. Fedotov, A. V. Talyzin, I. Suarez Lopez, I. V. Anoshkin, A. G.

Nasibulin, E. I. Kauppinen, E. D. Obraztsova, ACS Nano 2013, 7, 6346.

[8] A.V.Talyzin, S. M. Luzan, K. Leifer, S. Akhtar, J. Fetzer, F. Cataldo, Y. O. Tsybin, C. W.

Tai, A. Dzwilewski, E. Moons, J. Phys. Chem. C 2011, 115, 13207.

[9] H. E. Lim, Y. Miyata, R. Kitaura, H. Kataura, H. Shinohara, 2012, APS March Meeting,

Boston, MA, http://meetings.aps.org/link/BAPS.2012.MAR.W6.1.

[10] B. Botka, M. E. Füstös, G. Klupp, D. Kocsis, M. Utczás, E. Székely, B. Simándi, Á.

Botos, R. Hack1, K. Kamarás, Phys. Status Solidi B 2012, 249, 2432.

[11] J. Xiao, H. Yang, Z. Yin, J. Guo, F. Boey, H. Zhang, Q. Zhang, J. Mater. Chem. 2011, $21,1423$.

[12] T. W. Chamberlain, J. Biskupek, G. A. Rance, A. Chuvilin, T. J. Alexander, E.

Bichoutskaia, U. Kaiser, A. N. Khlobystov, ACS Nano 2012, 6, 3943.

[13] M. Yudasaka, K. Ajima, K. Suenaga, T. Ichihashi, A. Hashimoto, S. Iijima, Chem. Phys.

Lett. 2003, 380, 42.

[14] F. Simon, H. Kuzmany, H. Rauf, T. Pichler, J. Bernardi, H. Peterlik, L. Korecz, F. Fülöp, A. Jánossy, Chem. Phys. Lett. 2004, 383, 362.

[15] A. N. Khlobystov, D. A. Britz, J. Wang, S. A. O'Neil, M. Poliakoff, G. A. D. Briggs, J. Mater. Chem. 2004, 14, 2852.

[16] J. Gao, P. Blondeau, P. Salice, E. Menna, B. Bártová, C. Hébert, J. Leschner, U. Kaiser, M. Milko, C. Ambrosch-Draxl, M. A. Loi, Small 2011, 7, 1807. 
[17] S. Reich, C. Thomsen, J. Maultzsch, Carbon Nanotubes: Basic Concepts and Physical

Principles, Wiley-VCH, Weinheim, Germany, 2004.

[18] Á. Botos, A. N. Khlobystov, B. Botka, R. Hackl, E. Székely, B. Simándi, K. Kamarás, Phys. Status Solidi B 2010, 247, 2743.

[19] B. Botka, Á. Pekker, Á. Botos, K. Kamarás, R. Hackl, Phys. Status Solidi B 2010, 247, 2843.

[20] M. J. O'Connell, S. M. Bachilo, C. B. Huffmann, V. C. Moore, M. S. Strano, E. H.

Haroz, K. L. Rialon, P. J. Boul, W. H. Noon, C. Kittrell, J. Ma, R. H. Hauge, R. B. Weissman, R. E. Smalley, Science 2002, 297, 593.

[21] H. J. Lempka, S. Obenland, W. Schmidt, Chem. Phys. 1985, 96, 349.

[22] H. Muramatsu, T. Hayashi, Y. A. Kim, D. Shimamoto, M. Endo, V. Meunier, B. G. Sumpter, M. Terrones, M. S. Dresselhaus, Small 2009, 5, 2678.

[23] J. H. Kim, M. Kataoka, D. Shimamoto, H. Muramatsu, Y. C. Jung, T. Hayashi, Y. A. Kim, M. Endo, J. S. Park, R. Saito, M. Terrones, M. S. Dresselhaus, ACS Nano 2010, 4, 1060. [24] D. A. Tsyboulski, Y. Hou, N. Fakhri, S. Ghosh, R. Zhang, S. M. Bachilo, M. Pasquali, L. Chen, J. Liu, R. B. Weisman, Nano Lett. 2009, 9, 3282.

[25] Y. Miyata, M. Suzuki, M. Fujihara, Y. Asada, R. Kitaura, H. Shinohara, ACS Nano 2010, 4, 5807.

[26] M. A. Loi, J. Gao, F. Cordella, P. Blondeau, E. Menna, B. Bártová, C. Hébert, S. Lazar, G. A. Botton, M. Milko, C. Ambrosch-Draxl, Adv. Mater. 2010, 22, 1635.

[27] K. Setyowati, M. J. Piao, J. Chen, H. Liu, Appl. Phys. Lett. 2008, 92, 043105.

[28] S. Ben-Valid, B. Botka, K. Kamarás, L. Reiss, A. Zeng, S. Yitzchaik, Carbon 2010, 48, 2773.

[29] S. M. Bachilo, M. S. Strano, C. Kittrell, R. H. Hauge, R. E. Smalley, R. B. Weissman, Science 2002, 298, 2361. 
[30] S. M. Bachilo, L. Balzano, J. E. Herrera, F. Pompeo, D. E. Resasco, R. B. Weissman, J. Am. Chem. Soc. 2003, 125, 11186.

[31] R. Pfeiffer, C. Kramberger, F. Simon, H. Kuzmany, V. N. Popov, H. Kataura, Eur. Phys. J. B 2004, 42, 345.

[32] R. Pfeiffer, F. Simon, H. Kuzmany, V. N. Popov, Phys. Rev. B 2005, 72, 161404.

[33] S. Bandow, M. Takizawa, K. Hirahara, M. Yudasaka, S. Iijima, Chem. Phys. Lett. 2001, 337, 48.

[34] F. Simon, H. Kuzmany, Chem. Phys. Lett. 2006, 425, 85.

[35] H. Shiozawa, T. Pichler, A. Grüneis, R. Pfeiffer, H. Kuzmany, Z. Liu, K. Suenaga, H. Kataura, Adv.Mater. 2008, 20, 1443.

[36] M. Koshino, Y. Niimi, E. Nakamura, H. Kataura, T. Okazaki, K. Suenaga, S. Iijima, Nature Chem. 2010, 2, 117.

[37] R. Pfeiffer, M. Holzweber, H. Peterlik, H. Kuzmany, Z. Liu, K. Suenaga, H. Kataura, Nano Lett. 2007, 7, 2428.

[38] Á. Pekker, K. Kamarás, Phys. Rev. B 2011, 84, 075475.

[39] D. A. Britz, A. N. Khlobystov, Chem Soc. Rev. 2006, 35, 637. 


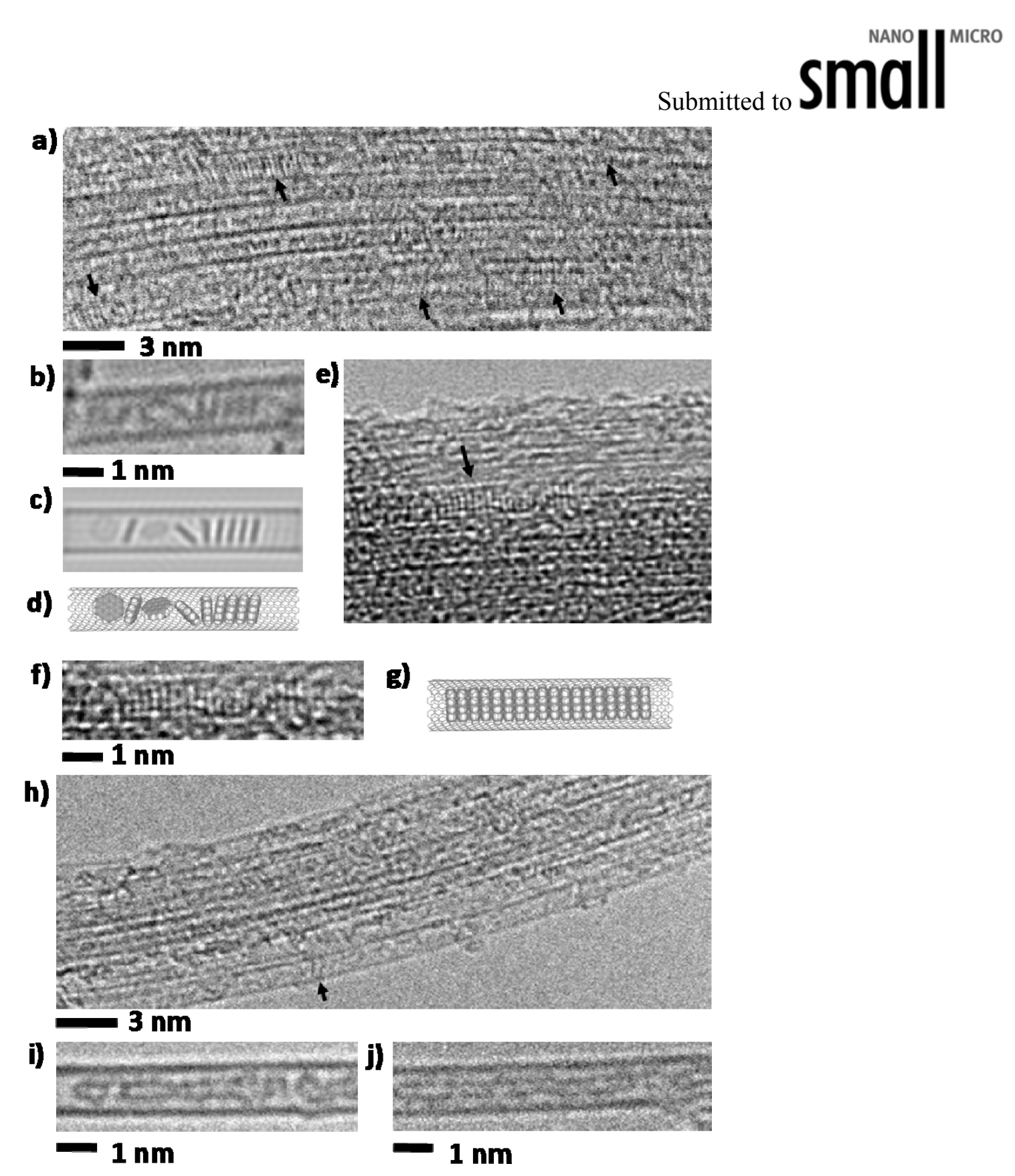

Figure 1. TEM images of P2-SWNTs filled with coronene at $50{ }^{\circ} \mathrm{C}$ using supercritical $\mathrm{CO}_{2}$ $(\mathrm{a}, \mathrm{b})$, and in the gas phase at $385^{\circ} \mathrm{C}(\mathrm{e}, \mathrm{f})$ or $450{ }^{\circ} \mathrm{C}(\mathrm{h})$. Short black arrows indicate the positions of coronene stacks in nanotubes within bundles. The structural diagram (d) and corresponding simulated TEM image (c) show how the coronene molecules stack inside the nanotube and are in good agreement with the experimental image (b). Stacks of coronene molecules are seen to extend through the nanotube interior, the structural diagram (g) corresponds to experimental image (f). Coronene molecules are unstable in the electron beam and therefore quickly transform into amorphous carbon or internal nanotube (i and j). 


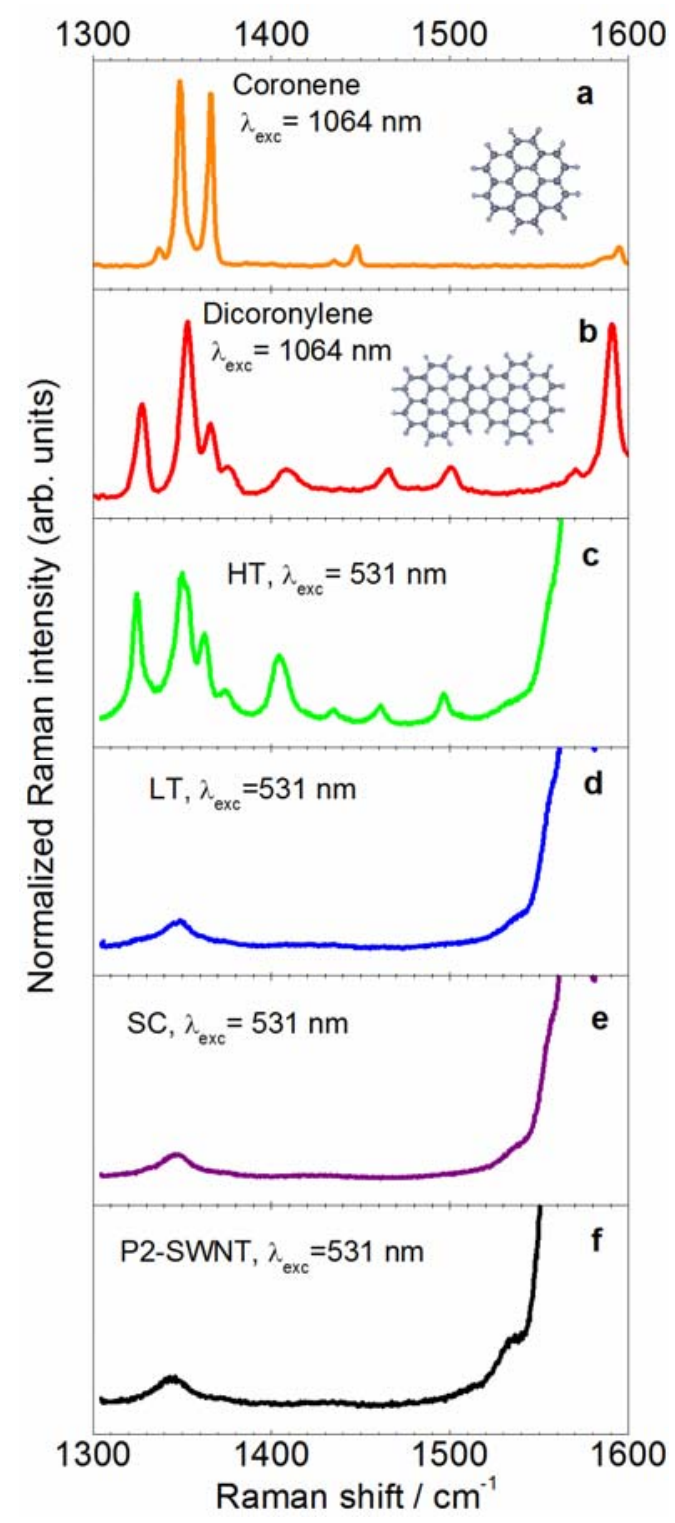

Figure 2. Raman spectra of P2-SWNT based and reference materials: (a) coronene, (b) dicoronylene, (c) Sample HT, (d) Sample LT, (e) Sample SC, (f) reference P2-SWNT. Spectra (c), (d), (e) and (f) are normalized to the nanotube G-band. 


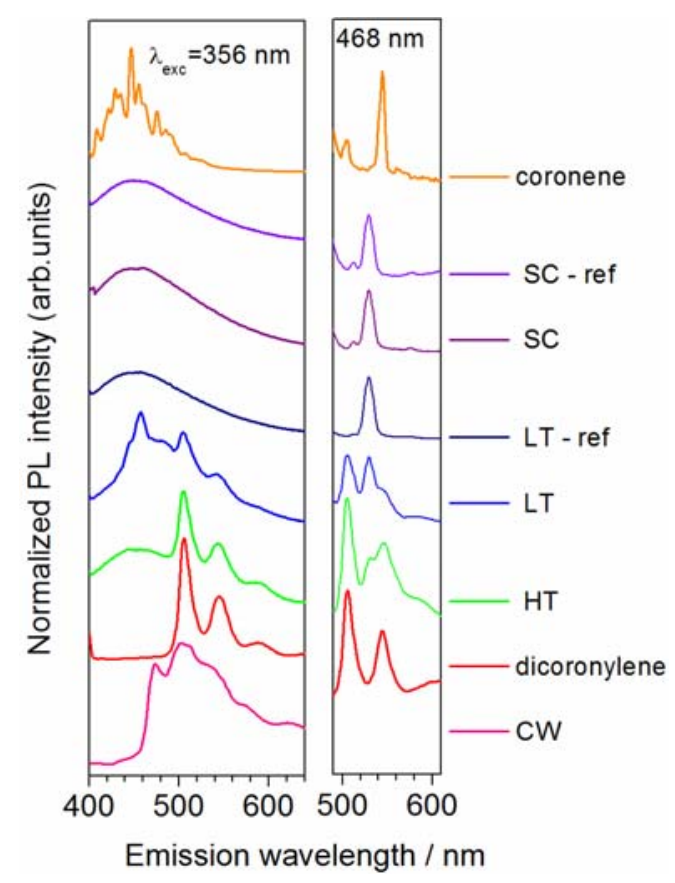

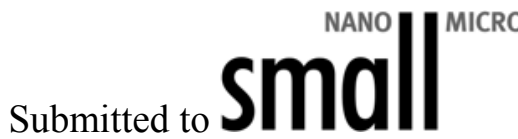

Figure 3. Photoluminescence spectra of coronene, dicoronylene in toluene, coronene nanowires (Sample CW), and samples SC, LT and HT in aqueous SDS solution at excitation wavelengths of $356 \mathrm{~nm}$ and $468 \mathrm{~nm}$. P2-SWNT samples which underwent identical treatment as samples SC and LT are marked as "ref". 
sunsus smäll
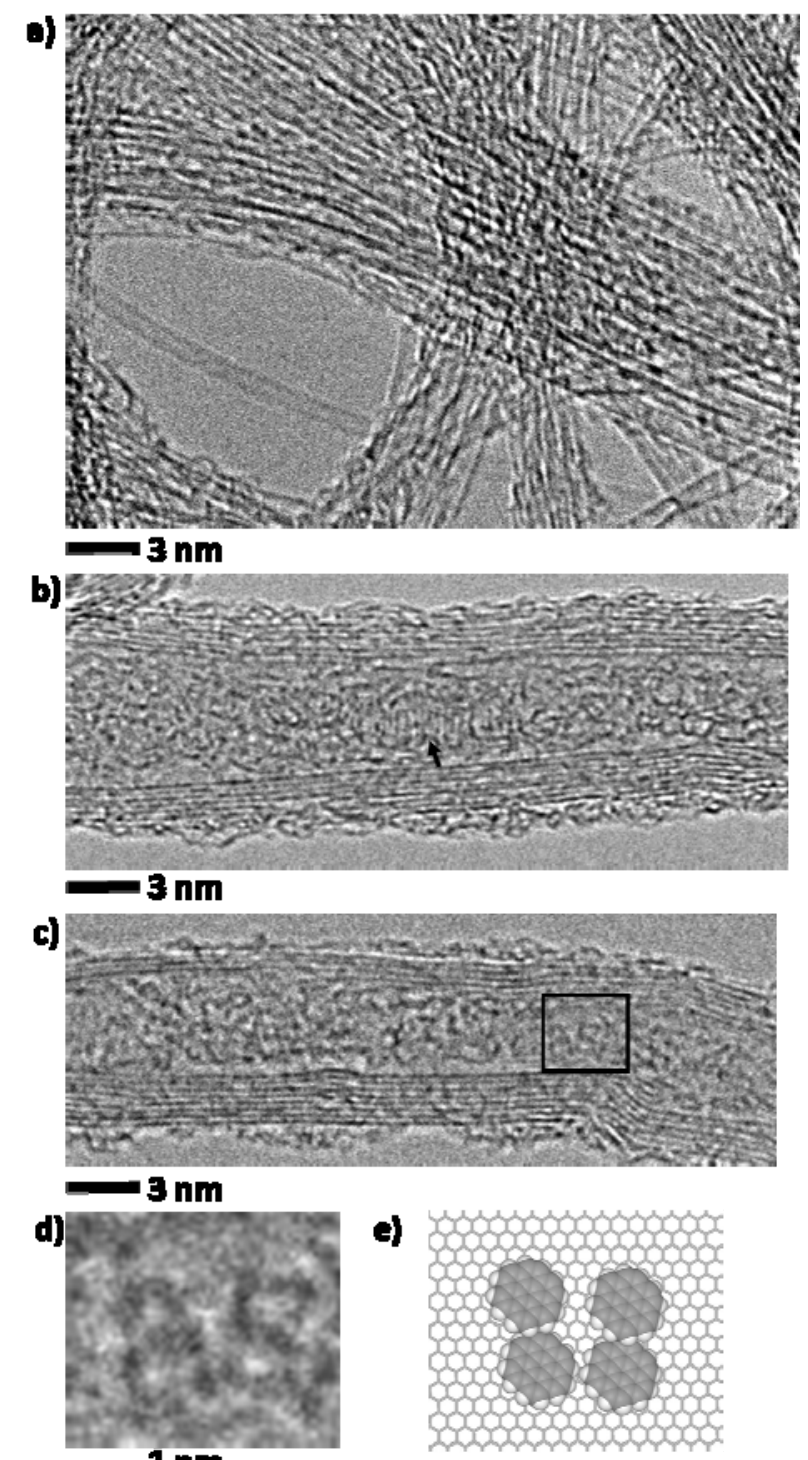

Figure 4. TEM images of CoMoCat-SWNT (a) and MWNT (b, c) exposed to coronene in the vapor phase at $450{ }^{\circ} \mathrm{C}$. The diameter of CoMoCat-SWNT is too small for coronene to enter into the cavity, so these nanotubes remain predominantly empty (a). The large internal diameter of MWNT encourages the formation of poorly ordered coronene structures $(b, c)$ with regular coronene stacks observed only rarely (black arrow, b). Because the molecules have greater rotational freedom in wider nanotubes, the formation of dicoronylene species is more likely in MWNT (c, the area within the black square is enlarged in d). The structural diagram (e) corresponds to a potential packing orientation of coronene molecules inside the wide nanotube (c and d). 
sunums smalli

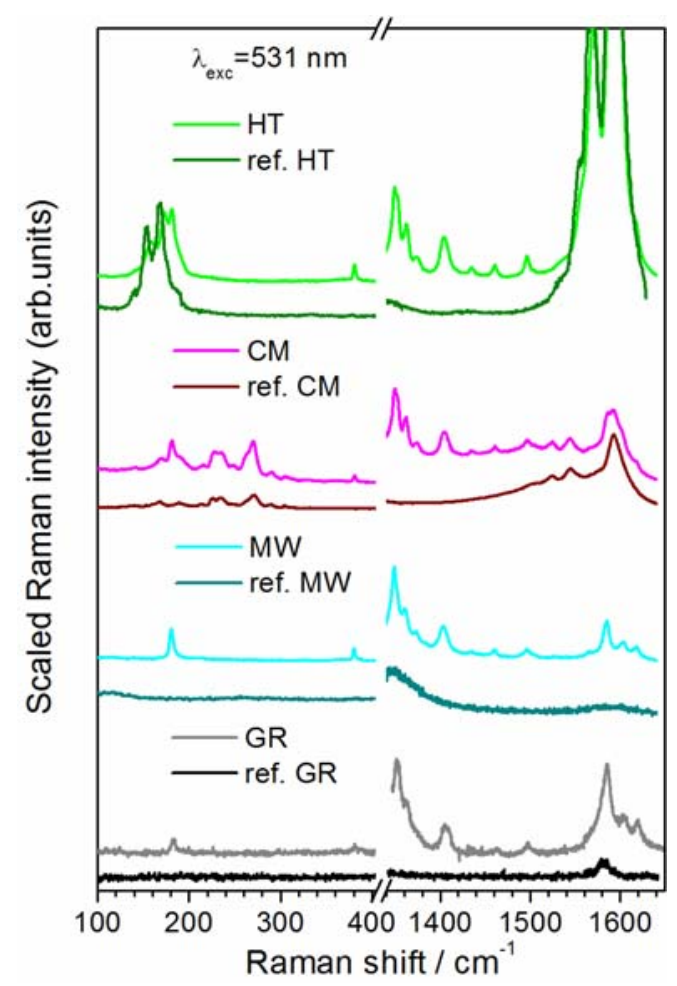

Figure 5. Raman spectra of samples HT, CM, MW and GR, and the corresponding reference materials. Reference materials are carbon structures which underwent the same thermal treatment as the coronene-carbon heterostructures, without applying coronene. The dicoronylene spectrum (Fig. 2b) appears in all samples. 


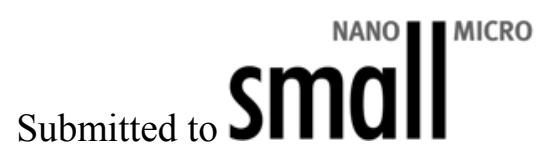

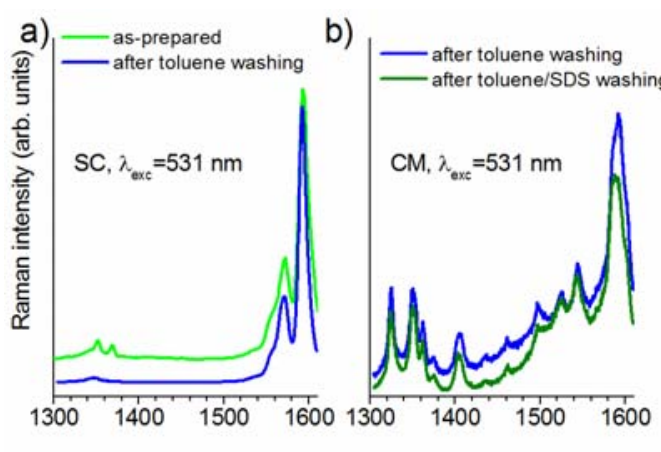

Raman shift $/ \mathrm{cm}^{-1}$

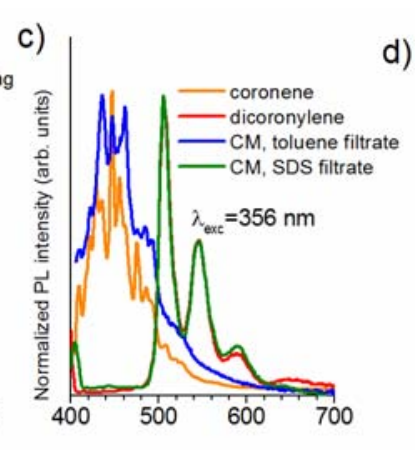

Emission wavelength / $\mathrm{nm}$ d)

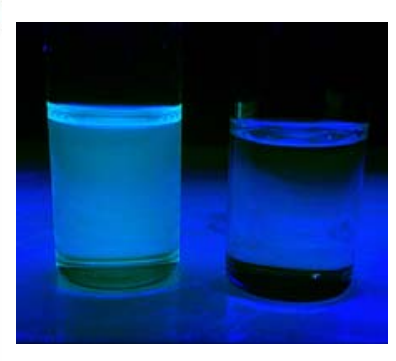

Figure 6. (a) Raman spectrum of Sample SC before and after washing with toluene. Raman bands of coronene disappear after washing. (b) Raman spectrum of Sample CM after washing with toluene and aqeous SDS solution. Dicoronylene Raman bands apparently do not lose intensity. All Raman spectra are normalized to the nanotube $\mathrm{G}$ band around $1600 \mathrm{~cm}^{-1}$. (c) Photoluminescence spectra of toluene and SDS filtrate of sample CM, compared to coronene and dicoronylene solutions in the respective solvents. Toluene filtrate contains coronene, SDS filtrate contains dicoronylene. (d) Photograph of the photoluminescence under UV lamp illumination (365 nm) of the first (left) and 3rd (right) aqueous SDS washing liquid of sample CM. (These are filtered solutions that contain only a minimal amount of nanotubes.) The washing was applied after toluene treatment. The first solution shows much stronger green fluorescence. 


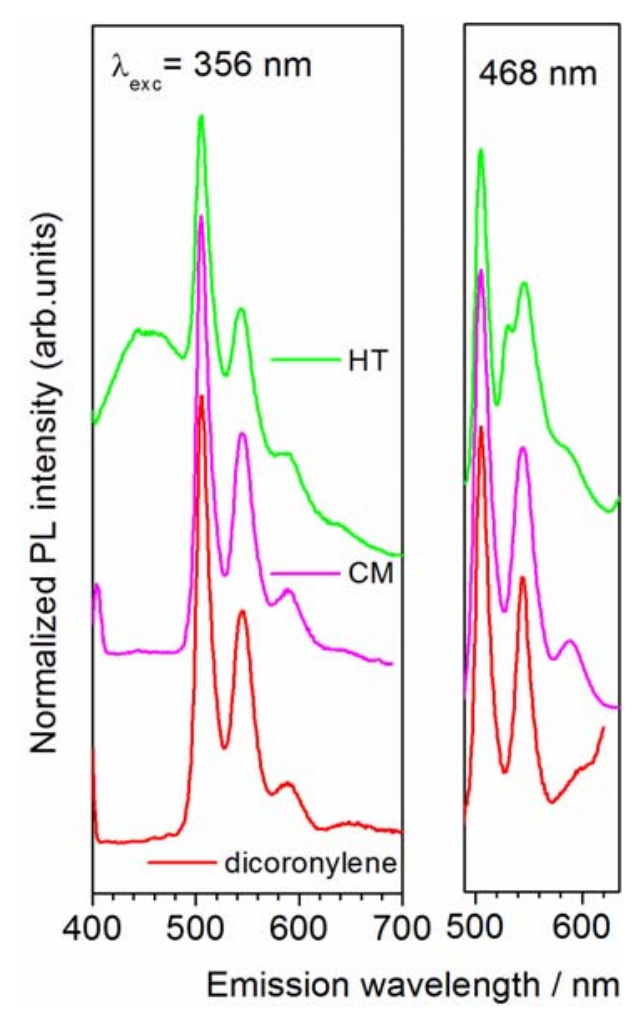

statisus small

Figure 7. Photoluminescence spectra of dicoronylene in toluene and samples HT and CM in aqueous SDS solution at excitation wavelengths $356 \mathrm{~nm}$ and $468 \mathrm{~nm}$. The broad low frequency band in the $356 \mathrm{~nm}$ HT spectrum and the $529 \mathrm{~nm}$ band in the $468 \mathrm{~nm}$ HT spectrum are nanotube features, see Fig. 3. 


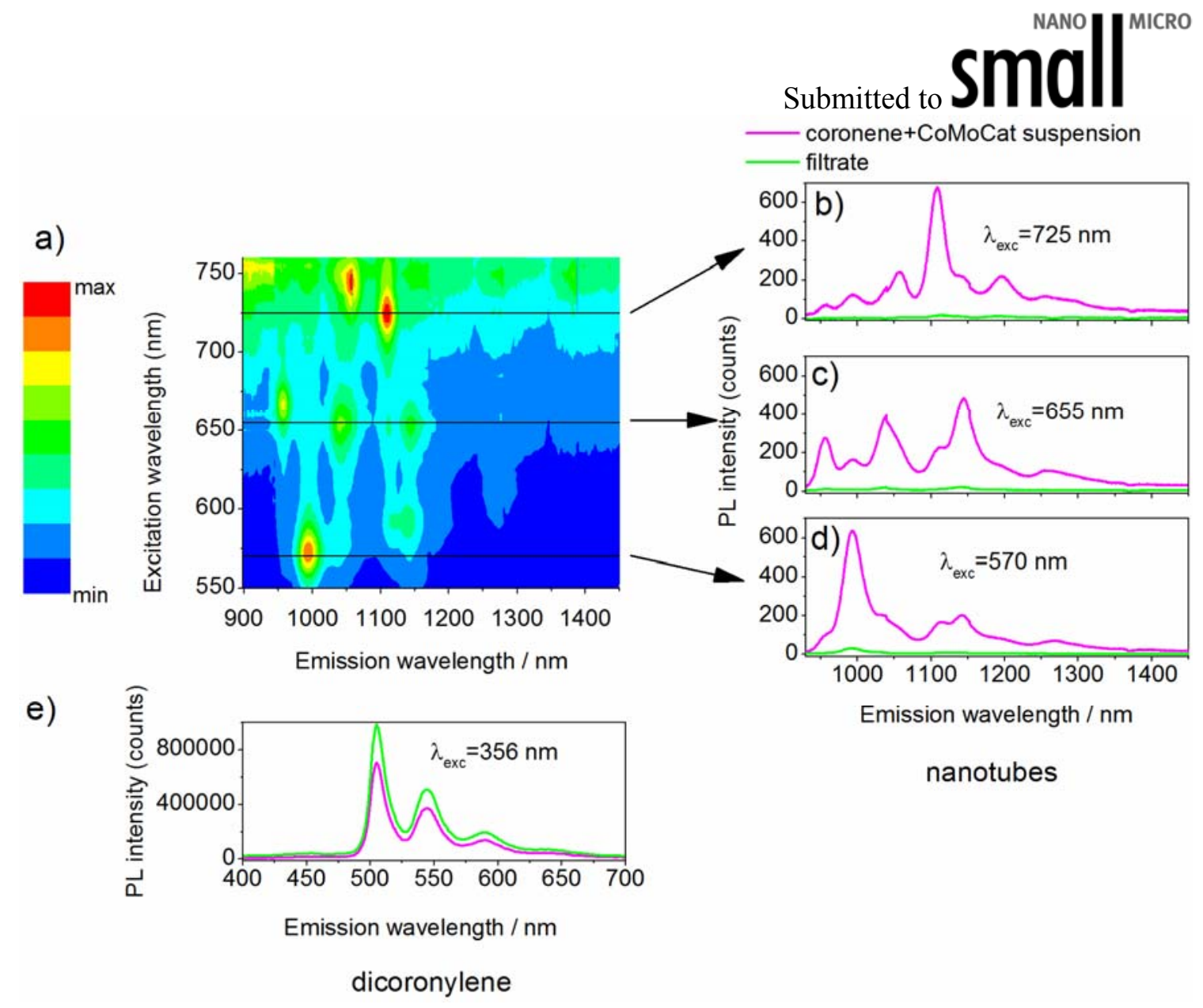

Figure 8. (a): Photoluminescence excitation map of sample CM (CoMoCat-SWNT), (b,c,d): photoluminescence of the suspension containing nanotubes and the filtrate, at selected NIR excitation wavelengths, (e): photoluminescence of the suspension and the filtrate at $356 \mathrm{~nm}$, the excitation wavelength used for dicoronylene. The filtrate contains only a negligible amount of nanotubes but shows strong luminescence in the visible, due to dissolved dicoronylene. 


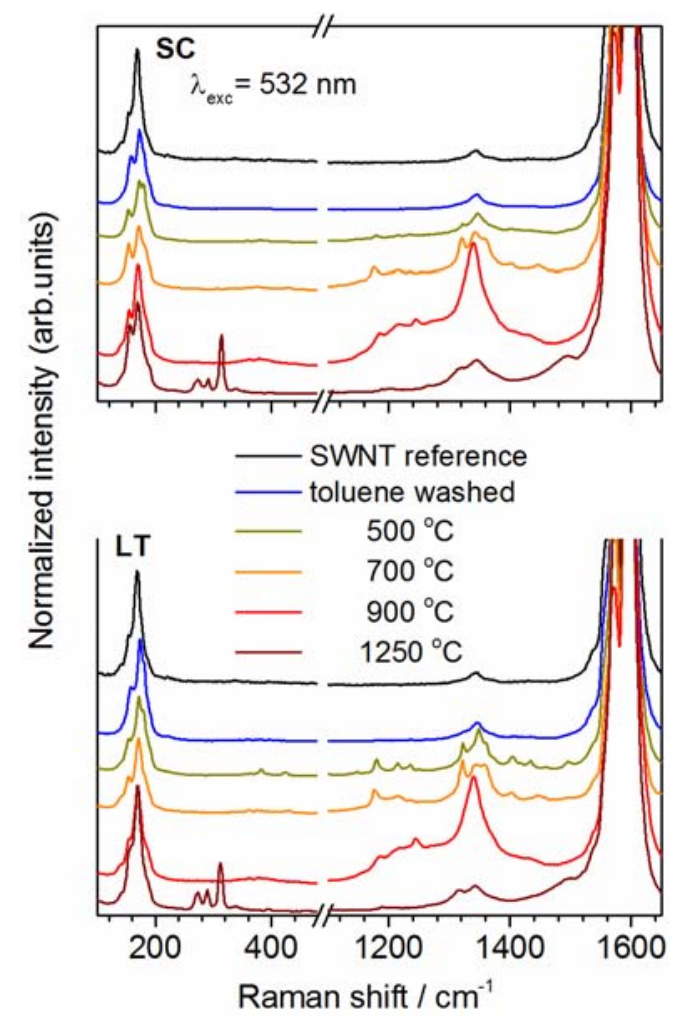

statisus small

Figure 9. Effect of annealing on Raman spectra of sample SC and LT showing the growth process of the inner nanotube. 


\section{sandestsmall}

Table 1. Summary of the different carbon structures and experimental conditions to form coronene/carbon nanostructure heterostructures.

\begin{tabular}{|c|c|c|c|c|c|}
\hline Sample & $\begin{array}{l}\text { Carbon } \\
\text { structure }\end{array}$ & Manufacturer & $\begin{array}{c}d \\
{[\mathrm{~nm}]}\end{array}$ & $\begin{array}{l}\text { Reaction } \\
\text { type }\end{array}$ & $\begin{array}{c}\mathrm{T} \\
{\left[{ }^{\circ} \mathrm{C}\right.}\end{array}$ \\
\hline $\begin{array}{l}\text { SC } \\
\text { (SuperCritical) }\end{array}$ & $\mathrm{P} 2$ & Carbon Solutions & 1.4 & $\mathrm{sc}-\mathrm{CO}_{2}$ & 50 \\
\hline $\begin{array}{l}\text { LT } \\
\text { (Low Temperature) }\end{array}$ & $\mathrm{P} 2$ & Carbon Solutions & 1.4 & Vapor & 385 \\
\hline $\begin{array}{l}\text { HT } \\
\text { (High Temperature) }\end{array}$ & $\mathrm{P} 2$ & Carbon Solutions & 1.4 & Vapor & 450 \\
\hline $\begin{array}{l}\mathrm{CM} \\
\text { (CoMoCat) }\end{array}$ & $\begin{array}{l}\text { CoMoCat } \\
\text { CG }\end{array}$ & $\begin{array}{c}\text { Southwest } \\
\text { Nanotechnologies }\end{array}$ & 0.9 & Vapor & 450 \\
\hline $\begin{array}{l}\text { MW } \\
\text { (Multi-Walled) }\end{array}$ & Ox-MWNT & Nanocyl & $4 @ 9^{a)}$ & Vapor & 450 \\
\hline $\begin{array}{l}\text { GR } \\
\text { (Graphite) }\end{array}$ & Graphite & Johnson Matthey & - & Vapor & 450 \\
\hline $\begin{array}{l}\mathrm{CW} \\
\text { (Coronene NanoWire) }\end{array}$ & $\begin{array}{l}\text { Coronene } \\
\text { nanowires }\end{array}$ & after Ref. [11] & - & Solution & 25 \\
\hline
\end{tabular}

${ }^{\text {a) }}$ Smallest inner and largest outer diameter.

Table 2. Composition, structure and transformation of the coronene/carbon nanostructure heterostructures investigated in this study. (In the table, "cor" stands for coronene and "dicor" for dicoronylene.)

\begin{tabular}{lccccc}
\hline Sample & $\begin{array}{c}\text { Carbon } \\
\text { structure }\end{array}$ & Outside & Inside & Comment & High T/e- beam \\
\hline SC & P2 & cor & cor & ordered stacks & polymer, DWNT \\
LT & P2 & cor + some dicor & cor & partly ordered stacks & polymer, DWNT \\
HT & P2 & cor + dicor & mixture & & polymer, DWNT \\
CM & CoMoCat CG & cor + dicor & no & & N/A \\
MW & Ox-MWNT & cor + dicor & mixture & & dimer \\
GR & Graphite & cor + dicor & N/A & & N/A \\
CW & Nanowire & N/A & N/A & ordered stacks & N/A \\
\hline
\end{tabular}




\section{sismutat small}

The table of contents entry should be 50-60 words long (max. 400 characters), and the first phrase should be bold. The entry should be written in the present tense and impersonal style. The text should be different from the abstract text.

Keyword coronene; graphene nanoribbons; carbon nanotubes; encapsulation

Bea Botka, Melinda E. Füstös, Hajnalka M. Tóháti, Katalin Németh, Gyöngyi Klupp, Zsolt Szekrényes, Dorina Kocsis, Margita Utczás, Edit Székely, Tamás Váczi, György Tarczay, Rudi Hackl, Thomas W. Chamberlain, Andrei N. Khlobystov, and Katalin Kamarás

Interactions and Chemical Transformations of Coronene Inside and Outside Carbon Nanotubes

ToC figure ((Please choose one size: $55 \mathrm{~mm}$ broad $\times 50 \mathrm{~mm}$ high or $110 \mathrm{~mm}$ broad $\times 20 \mathrm{~mm}$ high. Please do not use any other dimensions))

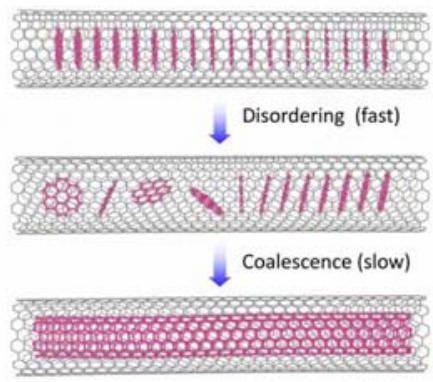


sunums smalli

Copyright WILEY-VCH Verlag GmbH \& Co. KGaA, 69469 Weinheim, Germany, 2013.

\section{Supporting Information}

for Small, DOI: 10.1002/smll.((please add manuscript number))

Interactions and Chemical Transformations of Coronene Inside and Outside Carbon Nanotubes

Bea Botka, Melinda E. Füstös, Hajnalka M. Tóháti, Katalin Németh, Gyöngyi Klupp, Zsolt Szekrényes, Dorina Kocsis, Margita Utczás, Edit Székely, Tamás Váczi, György Tarczay, Rudi Hackl, Thomas W. Chamberlain, Andrei N. Khlobystov, and Katalin Kamarás

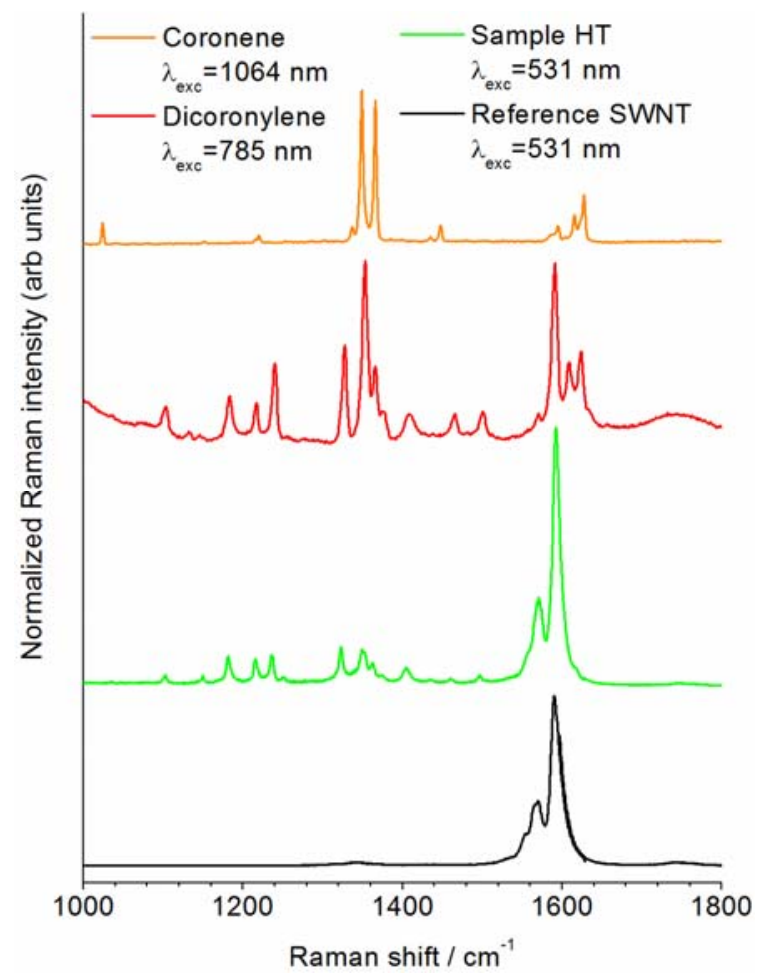

Figure S1. Raman spectra of the starting SWNT, coronene, dicoronylene and sample HT.

The spectrum of dicoronylene taken with $1064 \mathrm{~nm}$ excitation is identical to the one shown in the figure with $785 \mathrm{~nm}$ excitation. 


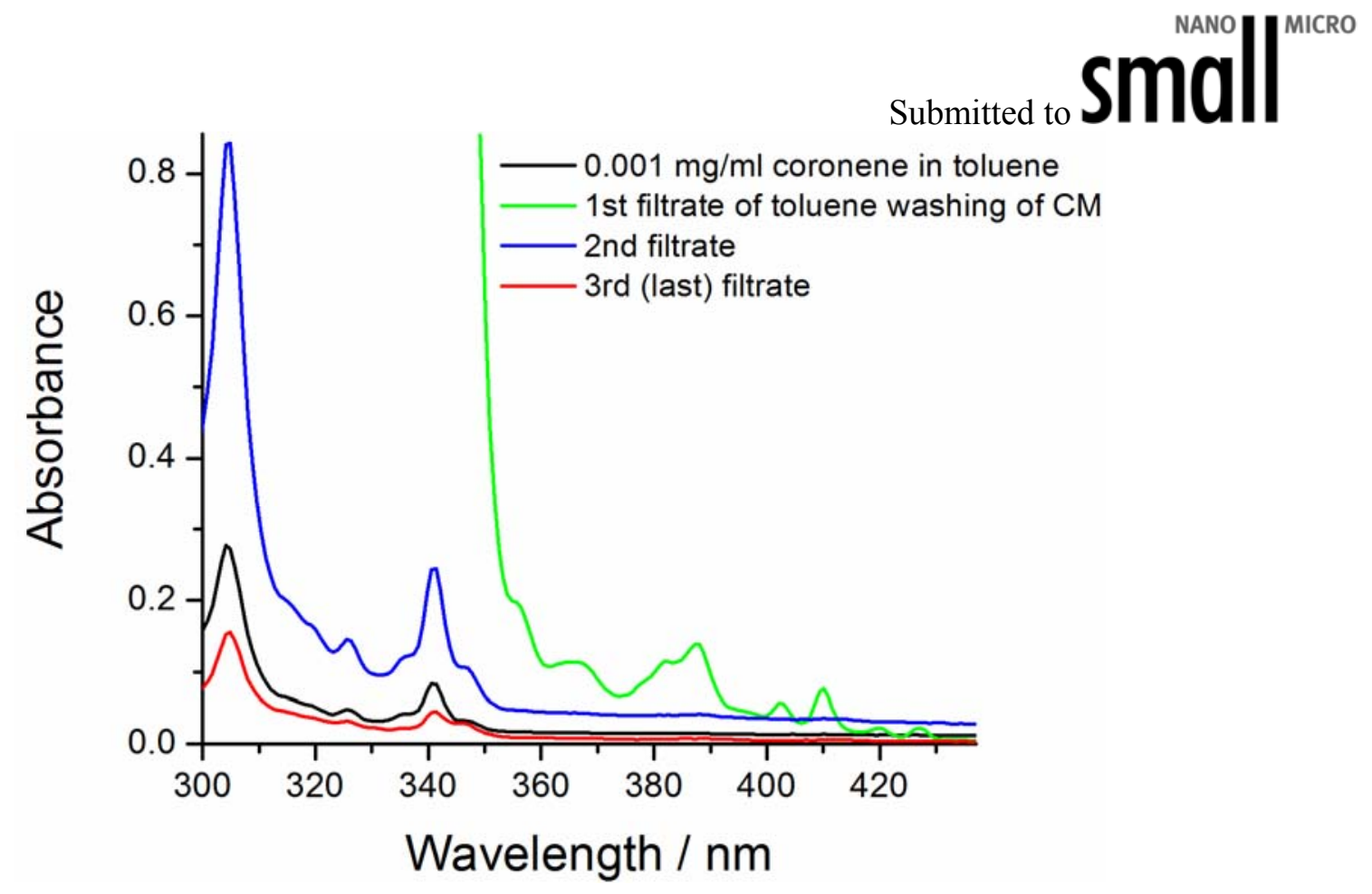

Figure S2. Absorption spectra of toluene filtrate solutions of the CM sample after the first, second and third filtering step. The last filtrate contains less than $0.001 \mathrm{mg} / \mathrm{ml}$ of coronene. 


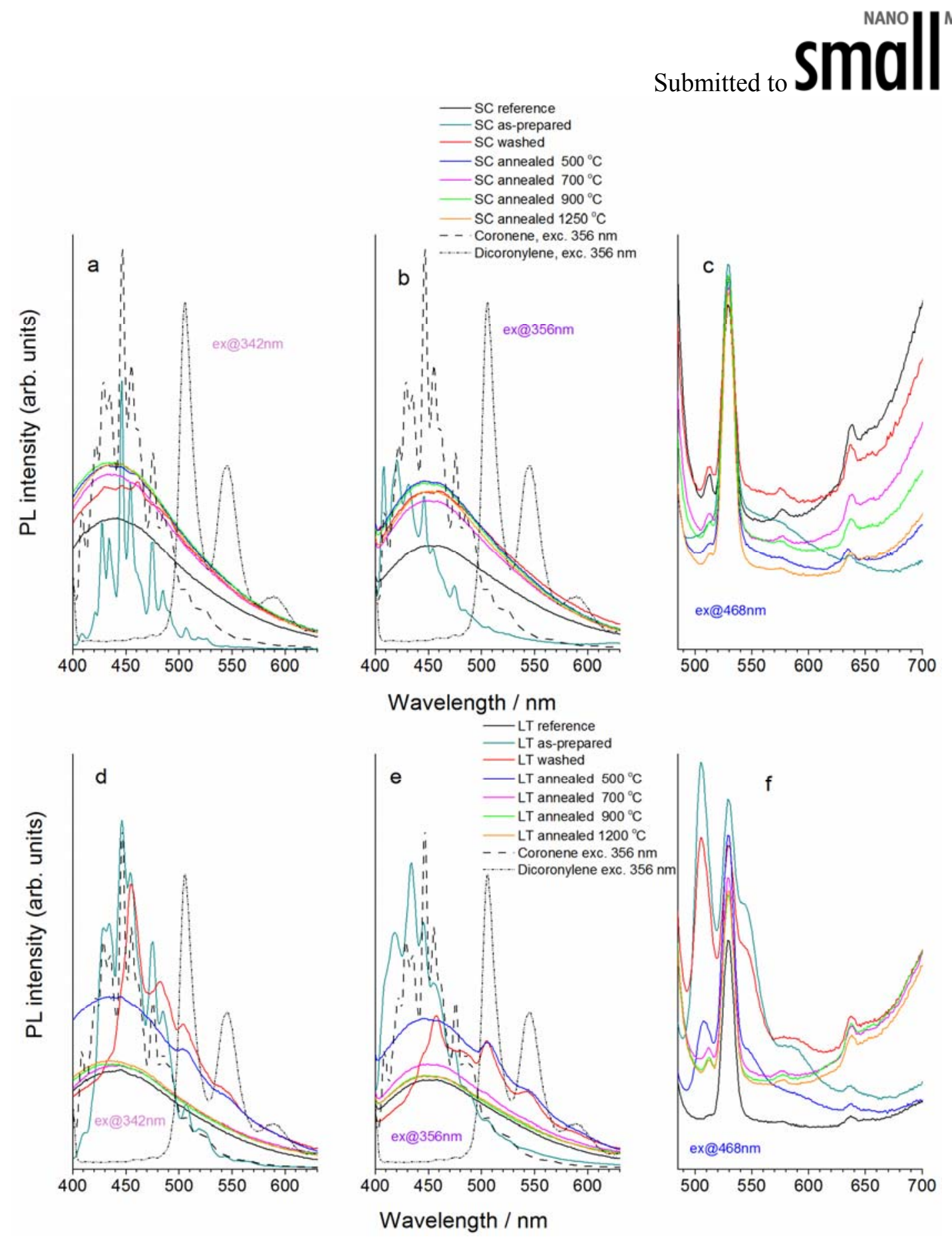

Figure S3. Effect of washing and annealing on photoluminescence spectra of sample SC (a-c) and LT (d-f). The SC sample, prepared at $50{ }^{\circ} \mathrm{C}$ and not containing polymerized coronene, loses PL intensity after washing with toluene. In contrast, sample LT prepared at $385{ }^{\circ} \mathrm{C}$ and containing a small amount of dicoronylene, retains the PL signal even after annealing at $500{ }^{\circ} \mathrm{C}$. None of the samples whose surface is "clean" show detectable photoluminescence by encapsulated species. (The background signal is caused by $\mathrm{D}_{2} \mathrm{O}$ and SDS.) 